\title{
The impact of COVID-19 on elective and urgent digestive endoscopic procedures: a report on a year of pandemic in a gastroenterology centre in Italy
}

\author{
Giovanna Del Vecchio Blanco ${ }^{1}$, Edoardo Troncone ${ }^{1}$, Enrico Grasso ${ }^{1}$, Elena De Cristofaro ${ }^{1}$, Giorgia Sena ${ }^{1}$, \\ Pasquale De Vico ${ }^{2}$, Mario Dauri ${ }^{2}$, Giovanni Monteleone ${ }^{1}$, Omero Alessandro Paoluzi ${ }^{1}$ \\ ${ }^{1}$ Gastroenterology Unit, Department of Systems Medicine, University of Rome “Tor Vergata”, Rome, Italy \\ ${ }^{2}$ Anaesthesiology Unit, Department of Clinical Sciences and Translational Medicine, University of Rome "Tor Vergata”, Rome, Italy
}

Gastroenterology Rev 2022; 17 (4): 301-309

DOI: https://doi.org/10.5114/pg.2021.111392

Key words: colonoscopy, COVID-19, endoscopic-retrograde-cholangio-pancreatography, endoscopic ultrasound, fine-needle aspiration, esophagogastroduodenoscopy, SARS-CoV-2.

Address for correspondence: Omero Alessandro Paoluzi, Gastroenterology Unit, Department of Systems Medicine, University of Rome “Tor Vergata", 00133 Rome, Italy, phone: +39 06 20900969, fax: +39 06 20904437, e-mail: omeroalessandro.paoluzi@ptvonline.it

\begin{abstract}
Introduction: The COVID-19 pandemic (COVID-19) affected digestive endoscopic activity worldwide. Resumption and maintenance of elective endoscopic activity are crucial to containing the impact of COVID-19 on mortality and prognosis of gastrointestinal disorders, primarily cancers.

Aim: To assess the impact of COVID-19 during and after the lockdown period on endoscopic activity.

Material and methods: The endoscopic activity undertaken during the COVID-19-related lockdown (March 2020-May 2020) and in the post-lockdown period (June 2020-March 2021) was compared with that in the corresponding periods of the year before COVID-19 in a gastroenterology centre in Italy.

Results: During the lockdown period, there was a reduction in esophagogastroduodenoscopy (EGD), colonoscopy (CSPY), endoscopic ultrasound (EUS), and endoscopic-retrograde cholangiopancreatography (ERCP) of $75.8 \%, 74.8 \%, 60 \%$, and $42 \%$, respectively, compared with the corresponding period of the year before COVID-19. During the post-lockdown period to date, EGD, CSPY, EUS, and ERCP increased as compared to the lockdown period (30.6\%, 50.6\%, 33.6\%, and 65.4\%, respectively), but only ERCP showed a full recovery when compared with the corresponding period of the year before COVID- 19 .

Conclusions: Endoscopic activity decreased significantly during the COVID-19 lockdown, and only ERCP had a full recovery in the post-lockdown period. The pandemic-related limitations and the backlog of endoscopic procedures represent important reasons for the increased risk or delayed diagnosis of GI cancers.
\end{abstract}

\section{Introduction}

In February 2020, after the first cases of coronavirus disease 2019 (COVID-19) [1] due to severe acute respiratory syndrome coronavirus 2 (SARS-CoV-2) were diagnosed in Italy, a series of decrees progressively limited travelling and working activities. Restrictions peaked on 9 March, when a complete "lockdown" involved all the Italian regions. From 23 March 2020, the health strategies adopted to contain the outbreak led to a redistribution of healthcare personnel in almost all wards of Italian hospitals [2]. Indeed, during the first wave of COVID-19 (March-May 2020), the elective gastroenterology activities and endoscopic procedures decreased by $>60 \%$ up to $100 \%$, compared to the ordinary volume registered in the pre-COVID19 period, and were limited to emergencies and management of oncological diseases [2-5]. In contrast, a structured questionnaire-based, nationwide, cross-sectional survey study did not find a significant reduction in the number of endoscopic-retrograde cholangiopancreatography (ERCP) undertaken in March 2020 versus March 2019 in Italy [6]. Little is known about the endoscopic activity during the so-called "post-lockdown period". To cope with a possible excessive demand for endoscopies after the lockdown period, the European Society of Gastrointestinal Endoscopy (ESGE) in July 2020 issued guidance to prioritize endoscopic procedures during the 
restarting period while maintaining active measures to prevent the spread of SARS-CoV-2 [7]. However, many high-priority endoscopic procedures, such as ERCP and biliary-pancreatic endoscopic ultrasound (EUS), are performed under anaesthesia, which may have received severe limitations during the pandemic.

\section{Aim}

In this study, we compared the gastro-intestinal endoscopic activities and the ERCP and EUS procedures during the COVID-19-related lockdown (March 2020May 2020) and in the post-lockdown period (June 2020-March 2021) with those in the corresponding periods of the year before COVID-19 in a gastroenterology centre in Italy.

\section{Material and methods \\ Changes related to the COVID-19 outbreak}

In March 2020, the Policlinico Tor Vergata, the hospital of the University of Rome "Tor Vergata", became a "COVID-19 Hospital", and its activity changed greatly. From 23 March to early June, the Emergency room was closed to non-COVID-19 patients except those with urgent or life-threatening conditions, all the internal medicine wards were dedicated to patients infected by SARS-CoV-2 except our Gastroenterology and Endoscopy Unit, and all elective surgery activity was relocated in other hospitals. Despite the operational status of our ward, following the indications by the Italian Council of Ministers to reduce the risk of spread of infection and contagion, from the beginning of the lockdown, we prioritized elective endoscopies already programmed in outpatients. Indications for a nondeferrable elective esophagogastroduodenoscopy (EGD) or colonoscopy (CSPY) in outpatients were the presence of alarm symptoms (weight loss, anorexia, anaemia, dysphagia, rectal bleeding, etc.) or higher risk of gastrointestinal cancer based upon previous clinical examination or a positive faecal occult blood test (FOBT), while non-alarm symptoms (e.g. reflux disease, dyspepsia, gastritis, Helicobacter pylori, abdominal pain, constipation, etc.) were considered not relevant and endoscopy was postponed. Endoscopies in inpatients were undertaken when deemed as: (i) urgent, due to an active bleeding (melena, hematemesis, massive rectal bleeding), impaction of foreign bodies, or other conditions requiring a mandatory endoscopic investigation within a few hours; or (ii) nondeferrable, being necessary for the diagnosis and/or treatment of the condition that required hospitalization. Indications for diagnostic endoscopic ultrasound (EUS) were choledocholithiasis, pancreatitis, pancreatic cysts or intraductal papillary mucinous neo- plasm (IPMN), radiological imaging raising the suspicion of a neoplasm of the upper digestive tract (e.g. gastrointestinal stromal tumour (GIST)), pancreas or biliary tract, staging of oesophageal or rectal cancer, or perianal fistula in patients affected by Crohn's disease with an abscess; we carried out a fine-needle aspiration during EUS (EUS-FNA) when a tissue diagnosis of tumour was necessary. Indications for ERCP were choledocholithiasis, stenosis of biliary tract due to pancreatic cancer or cholangiocarcinoma, insertion or substitution of a stent in patients with post-surgery leak after liver transplantation or surgery or with biliary stenosis.

After the end of the lockdown, the Emergency Room returned to open access for all patients. From early June 2020, the hospital management established a gradual restarting of endoscopic activities for outpatients, but the number of slots available for reservation through the National Health System (NHS) was limited in fear of having to tackle a new wave of infection. Relocation of nurses $(7 / 13,54 \%)$ from the Endoscopy Unit to the COVID-19 wards also limited the slots because it led to the reduction of operative endoscopic rooms from 4 to 1 . Considering the available slots, we re-evaluated the indication and appropriateness of the prescription of the endoscopies cancelled during the lockdown by telephone interview of the patients, and rescheduling was offered according to ESGE position statements on gastrointestinal endoscopy during the COVID-19 pandemic $[7,8]$. Colonoscopy for cancer prevention in patients with inflammatory bowel diseases (long-standing ulcerative colitis and Crohn's disease), surveillance after surgery for colorectal cancer, and post-polypectomy surveillance were restarted in keeping with ESGE guidelines $[9,10]$. Since the end of July 2020, our Endoscopy Unit was contacted by doctors in neighbouring hospitals asking to send us inpatients who needed to perform ERCP and EUS due to the interruption of their endoscopic activity or unavailability of their usual reference Endoscopy Unit. Before each procedure in such referrals, we received clinical information by direct contact with the colleague by phone and all clinical and radiological data (clinical history, laboratory tests, cholangio-RMN and/or CT scan) by mail or fax.

ERCP, EUS, and procedures with a surgical approach, like EGD with percutaneous endoscopic gastrostomy (EGDS-PEG) are undertaken under anaesthesia, while diagnostic EGD and CSPY under conscious sedation or, when available, under anaesthesia if requested by the patient or when necessary.

From the beginning of the COVID-19 pandemic, to prevent SARS-CoV-2 spread and contagion, in agreement with ESGE-EGENA guidance [8], we drafted a local procedure [11] that included the following: (i) prohibit- 
ing access to patient caregivers, a triage for risk stratification of SARS-CoV-2 infection in outpatients; (ii) the strict adherence to hygiene procedures and the use of personal protection equipment by doctors and nurses; and (iii) the reinforcement of the hygiene procedures in the endoscopy room after each investigation.

\section{Endpoints, collection of data, and statistical analysis}

The primary endpoint of the study was to evaluate the endoscopic activity defined as the number of endoscopic procedures performed. The secondary endpoint was the spread of SARS-CoV-2 infection among the staff of the Endoscopy Unit and in patients undergoing a digestive endoscopy.

In a retrospective manner, we reviewed all the endoscopic activity carried out from 23 March 2019 to 22 March 2021 in our Gastroenterology and Endoscopy Unit. Using specific queries, we selected in the electronic database (Endobase ${ }^{\circledR}$, Olympus Italia, Italy) used in our endoscopic sessions all the procedures, namely CSPY, EGD, EGD-PEG, ERCP, EUS, and EUS-FNA. Once selected, the endoscopic procedures were stratified according to patient status (outpatients or inpatients) and timing (elective, urgent, or nondeferrable). The endoscopic activity carried out during COVID-19-related lockdown (23 March 2020 to 18 May 2020) and in the post-lockdown period (19 May 2020 to 22 March 2021) was compared with the corresponding periods of the year before COVID-19. Data were analysed using IBM SPSS Statistics for Windows, Version 21.0. (IBM Corp., Armonk, N.Y., USA) and evaluated by univariate analysis. Statistical significance was determined using Student's $t$-test and the $\chi^{2}$ test. A $p$-value of $<0.05$ was considered statistically significant.

The spread of SARS-CoV-2 infection was defined based on COVID-19 cases among the staff of the Endoscopy Unit and in patients submitted to digestive endoscopy.

\section{Results}

\section{Overall changes in endoscopic activity} during the COVID-19 pandemic

The cumulative figures regarding different endoscopic procedures performed during the first 12 months of COVID-19 and in the corresponding period of the year before COVID-19 are shown in Table I. Overall, endoscopic activity during COVID-19 decreased by $44 \%$ compared to the previous year. The greatest drop occurred in elective endoscopy in outpatients, with a reduction of $71.6 \%$ and $42.2 \%$ in EGD and CSPY, respectively $(p<0.01)$. Nondeferrable EGD and CSPY in

Table I. Endoscopic procedures in outpatients and inpatients during the first year of COVID-19 compared with the pre-COVID-19 year

\begin{tabular}{|c|c|c|c|c|c|c|c|c|c|c|}
\hline \multirow[t]{2}{*}{ Procedures } & \multirow[t]{2}{*}{ Timing } & \multirow[t]{2}{*}{ Setting } & \multicolumn{3}{|c|}{ Pre-COVID-19 } & \multicolumn{3}{|c|}{ COVID-19 } & \multirow{2}{*}{$\begin{array}{c}\text { Change } \\
\text { (\%) }\end{array}$} & \multirow[t]{2}{*}{$P$-value } \\
\hline & & & All & $\begin{array}{c}\text { *23 Mar- } \\
18 \text { May } \\
2019\end{array}$ & $\begin{array}{c}\text { **19 May } \\
2019- \\
22 \text { Mar } \\
2020\end{array}$ & All & Lockdown & $\begin{array}{c}\text { Post- } \\
\text { lockdown }\end{array}$ & & \\
\hline \multirow[t]{4}{*}{$\mathrm{EGD}, n$} & All & Out/Inpatients & 2505 & 782 & 1723 & 1133 & 189 & 944 & -54.8 & $<0.01$ \\
\hline & Urgent & Inpatients & 178 & 38 & 140 & 143 & 18 & 125 & -19.6 & 0.05 \\
\hline & Elective & Outpatients & 1362 & 499 & 863 & 388 & 36 & 352 & -71.6 & $<0.01$ \\
\hline & Nondeferrable & Inpatients & 965 & 245 & 720 & 602 & 135 & 467 & -37.6 & $<0.01$ \\
\hline \multirow[t]{4}{*}{ CSPY, $n$} & All & Out/Inpatients & 2436 & 706 & 1730 & 1487 & 178 & 1309 & -39 & $<0.01$ \\
\hline & Urgent & Inpatients & 43 & 6 & 37 & 39 & 1 & 38 & -9.3 & 0.11 \\
\hline & Elective & Outpatients & 1770 & 517 & 1253 & 1023 & 32 & 991 & -42.2 & $<0.01$ \\
\hline & Nondeferrable & Inpatients & 623 & 183 & 440 & 425 & 145 & 280 & -31.8 & $<0.01$ \\
\hline EGDS-PEG, $n$ & Nondeferrable & Inpatients & 84 & 13 & 71 & 88 & 5 & 83 & 4.7 & 0.16 \\
\hline ERCP, $n$ & Nondeferrable & Inpatients & 157 & 50 & 107 & 161 & 29 & 132 & 2.5 & 0.23 \\
\hline \multirow[t]{3}{*}{ EUS, $n$} & All & Out/Inpatients & 349 & 95 & 254 & 225 & 38 & 187 & -35.5 & $<0.01$ \\
\hline & Elective & Outpatients & 143 & 38 & 105 & 102 & 29 & 73 & -28.6 & 0.42 \\
\hline & Nondeferrable & Inpatients & 206 & 57 & 149 & 123 & 9 & 114 & -40.2 & $<0.01$ \\
\hline
\end{tabular}

CSPY - colonoscopy, EGD - esophago-gastro-duodenoscopy, EGD-PEG - percutaneous endoscopic gastrostomy during EGD, ERCP - endoscopic-retrogradecholangiopancreatography, EUS - endoscopic ultrasound, EUS-FNA - EUS with fine-needle aspiration. *Pre-COVID-19 period corresponding to the COVID-19 lockdown. **Pre-COVID-19 period corresponding to the COVID-19 post-lockdown period. 
Table II. Numbers of endoscopic-retrograde cholangiopancreatography and endoscopic ultrasound during the first COVID-19 year and pre-COVID-19 year

\begin{tabular}{lccc} 
Procedures & Pre-COVID-19 & COVID-19 & Change (\%) \\
\hline ERCP, $n:$ & 157 & 161 & 2.5 \\
\hline Choledocholithiasis & 93 & 83 & -10.7 \\
\hline Malignancy of the pancreas or biliary tree & 26 & 38 & 46 \\
\hline Suspected stricture of biliary tract & 8 & 7 & -12.5 \\
\hline Post-liver transplant leak/stricture & 9 & 11 & 22 \\
\hline Stent for benign stricture & 21 & 22 & 4.7 \\
\hline EUS, $n:$ : & 349 & 225 & -35.5 \\
\hline Diagnostic without FNA: & 256 & 164 & -36 \\
\hline Cholestasis/jaundice & 58 & 40 & -31 \\
\hline Pancreatitis & 38 & 25 & -34.2 \\
\hline Pancreatic cysts/PMN & 36 & 23 & -36.1 \\
\hline Submucosal lesions of upper digestive tract & 23 & 15 & -34.7 \\
\hline Staging of oesophageal or rectal cancer & 19 & 12 & -36.8 \\
\hline Perianal fistula in patients with Crohn's disease & 26 & 19 & -26.9 \\
\hline Suspected choledocholithiasis & 36 & 17 & -52.7 \\
\hline Suspected stenosis of choledochus & 20 & 13 & -35 \\
\hline EUS with FNA, $n:$ & 93 & 61 & -34.4 \\
\hline Pancreas & 57 & 41 & -28 \\
\hline Biliary tract & 10 & 5 & -50 \\
\hline Stomach (GIST) & 3 & 7 & 233 \\
\hline Mediastinum & 15 & 5 & -80 \\
\hline Other & 85.5 & \\
\hline
\end{tabular}

ERCP - endoscopic-retrograde cholangiopancreatography, EUS - endoscopic ultrasound, FNA - fine-needle aspiration, GIST - gastrointestinal stromal tumour, IPMN - intraductal papillary mucinous neoplasm. *Pre-COVID-19 period corresponding to the COVID-19 lockdown. ${ }^{*}$ Pre-COVID-19 period corresponding to the COVID-19 post-lockdown period.

inpatients also diminished significantly, with a decrease of 37.6 and $31.8 \%$, respectively, whilst the urgent procedures remained unchanged. Nondeferrable EUS dropped by $35.5 \%(p<0.01)$, and ERCP remained unchanged. The indications for ERCP and EUS, with or without FNA, during the COVID-19 period showed variations but not significant in respect to the corresponding preCOVID-19 periods (Table II).

\section{The lockdown period is marked by decreased endoscopic activity}

Next, we examined the impact of the "lockdown period" on endoscopic activity. In line with previous findings, all the endoscopic procedures were markedly reduced during the lockdown period as compared to the activities performed in the corresponding months of the year before the COVID-19 pandemic. EGDS, CSPY, ERCP, and EUS decreased by $75.8 \%, 74.8 \%, 42 \%$, and $60 \%$, respectively, compared to those registered in the previous year $(p<0.01)$ (Figure 1$)$.

\section{Endoscopic procedures in the post-lockdown period}

Rescheduling of endoscopic procedures in outpatients, elective EGD and CSPY, cancelled during the lockdown period was accepted by $75 \%$ of them while the remaining $25 \%$ refused the proposal for fear of contracting the coronavirus while visiting the Endoscopy Unit. During the post-lockdown period, there was a significant increase in the endoscopic procedures, especially among outpatients, as compared to the lockdown period, with a gain of $30.6 \%, 50.5 \%, 33.6 \%$, and $65.4 \%$ in EGD, CSPY, EUS, and ERCP, respectively $(p<0.01)$. However, the total numbers of EGDS, CSPY, and EUS performed in the post-lockdown period were $45.2 \%, 24.3 \%$, and $26.4 \%$ lower than those registered in the corresponding period of the year before COVID-19, while the number of ERCP was significantly greater $(23.3 \%, p<0.01)$ (Figures 2 and 3$)$. Referrals from other hospitals accounted for $9.1 \%$ of the entire volume of biliopancreatic endoscopic procedures, 15\% of ERCP, 
and $4.9 \%$ of EUS. The collaboration network has grown gradually; to date we are the reference Endoscopy Unit for 6 neighbouring hospitals.

\section{Spread of SARS-CoV-2 among staff in the Endoscopy Unit}

Four out of 24 (17\%) healthcare workers in the Endoscopy Unit contracted SARS-CoV-2 infection, 2 developed a mild form of COVID-19 and 2 remained asymptomatic. A nurse and a doctor most likely had a familial infection, while 2 healthcare technicians probably contracted the infection by having contact with late-diagnosed COVID-19-infected inpatients. All cases occurred in the post-lockdown period. No nurse relocated in COVID-19 wards contracted infection, and no physician or nurse contracted infection by undertaking endoscopy in COVID-19 positive patients. No inpatient or outpatient developed COVID-19 following a procedure in our Endoscopy Unit.

\section{Discussion}

COVID-19 led to a significant decrease of the endoscopic activity in our Gastroenterology and Endoscopy Unit. As expected, the greatest impact of the outbreak occurred during the lockdown period, with a decrease of elective/nondeferrable procedures ranging from $\sim 42 \%$ to $72 \%$, in agreement with previous reports [36]. Urgent endoscopic procedures, mainly due to acute digestive bleeding, did not show a significant change. This suggests that our hospital continued to be a refer-

\section{A}

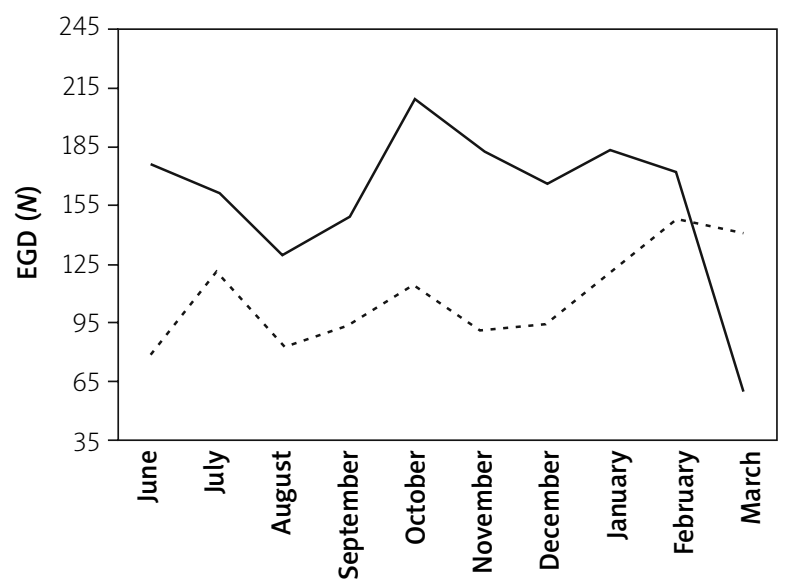

- Pre-COVID-19

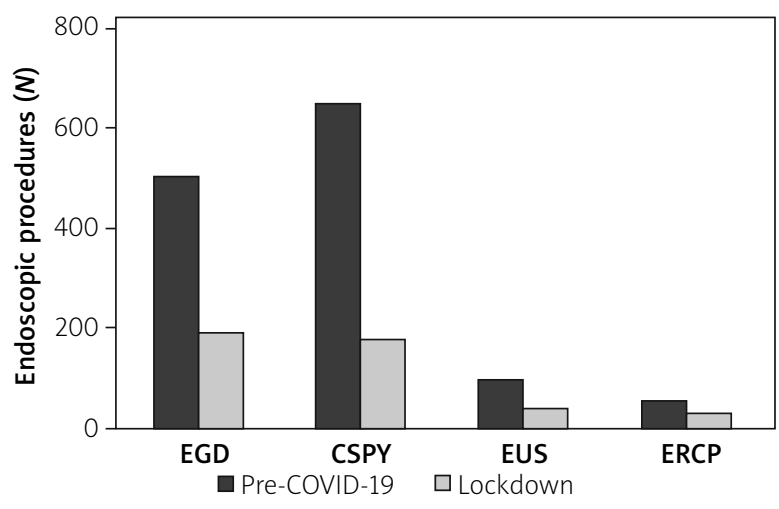

Figure 1. Endoscopic procedures carried out during COVID-19 pandemic-related lockdown (23 March 2020-18 May 2020) and in the corresponding period of the year before COVID-19. During lockdown, there was a marked decrease in the endoscopic activity, more pronounced in EGD and CSPY

CSPY - colonoscopy, EGD - esophago-gastro-duodenoscopy, $E R C P$ - endoscopic-retrograde cholangiopancreatography, EUS - endoscopic ultrasound.

ral for patients with life-threatening conditions, even though dedicated to receiving COVID-19 patients. Our finding is in contrast with a study from Austria, which reported a massive decrease of urgent upper digestive endoscopies for digestive bleeding during lockdown [12], and with a database analysis from the United Kingdom where urgent digestive endoscopies had an

\section{B}

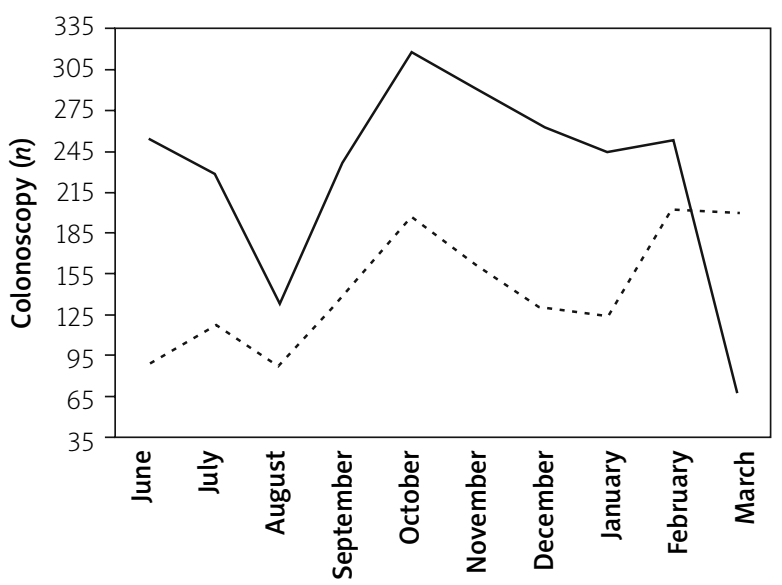

- - . Post-lockdown

Figure 2. Elective endoscopy in the COVID-19-related post-lockdown period (June 2020-March 2021) compared with the corresponding period of the year before COVID-19. Trends in the number of esophagogastroduodenoscopy (A) and colonoscopy (B) showed a gradual, but slight, recovery in comparison to the corresponding period in the year before COVID-19

CSPY - colonoscopy, EGD - esophago-gastro-duodenoscopy. 
A

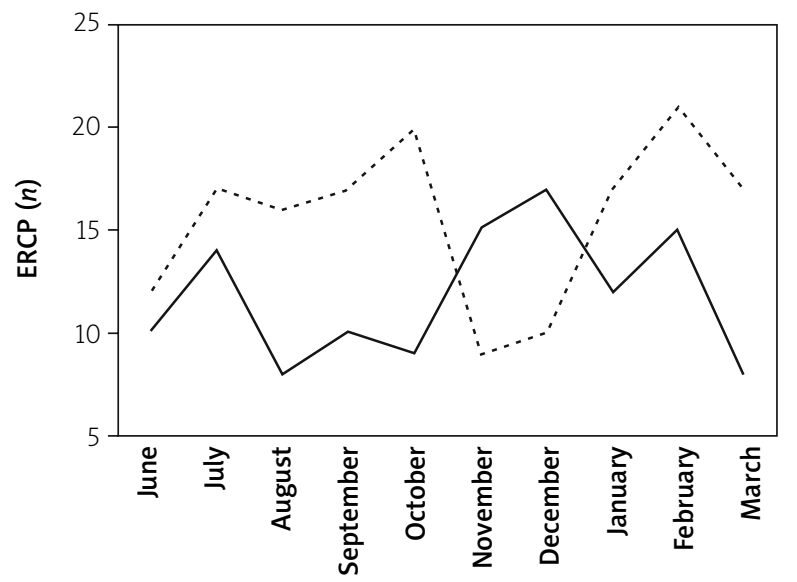

B

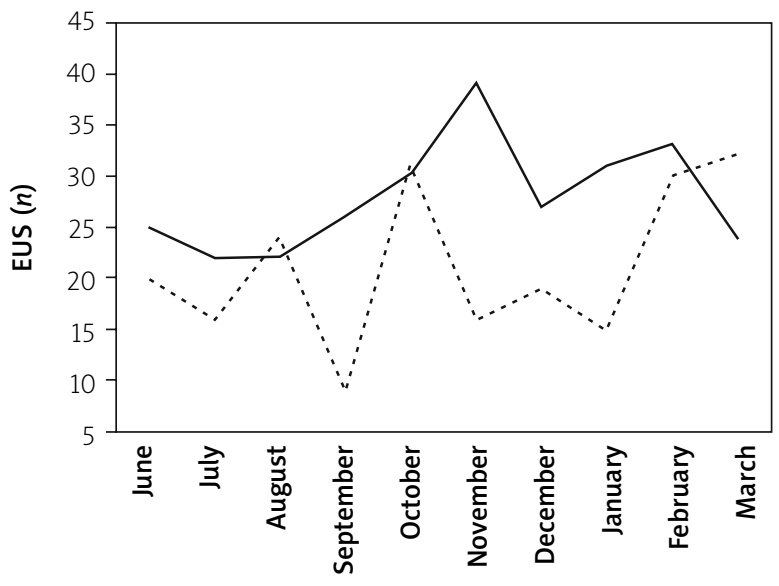

- Pre-COVID-19

-. - Post-lockdown

Figure 3. Endoscopic-retrograde cholangiopancreatography and endoscopic ultrasound undertaken in the COVID-19-related post-lockdown period (June 2020-March 2021) compared with the corresponding period of the year before COVID-19. Endoscopic-retrograde cholangiopancreatography had a significant rebound from June to October, exceeding the number of those performed in the same months in the pre-COVID-19 year (A), while endoscopic ultrasound had a slight but not significant recovery (B)

ERCP - endoscopic-retrograde cholangiopancreatography, EUS - endoscopic ultrasound.

increase from $28 \%$ to $57 \%$ [13]. The reasons of such a discrepancy are not clear.

During the post-lockdown period, the endoscopic procedures had only a partial and variable recovery. The numbers of EGD and CSPY in outpatients increased but, although they showed a similar monthly distribution, remained constantly below the figures in the corresponding period of the year before COVID-19. Our finding is in keeping with a study from the United Kingdom reporting that endoscopic activity in the COVID-19-impacted period reduced to $12 \%$ of pre-COVID-19 levels, recovering to $20 \%$ after the lockdown [13]. The partial resumption of endoscopic activity may have had various reasons in different countries. In our experience, a limitation was probably the partial reopening of our hospital, with limited slots for outpatients bookable through the NHS. On the other hand, when phoned, a quarter of the patients refused to reschedule the cancelled EGD and CSPY for fear of contracting the virus. A similar "fear-of-contagion" effect may also be considered responsible for the reduction in the endoscopies in the weeks immediately before the lockdown (Figure 2) when there were already frequent media reports of the overflow of COVID-19 patients in the hospitals. This finding suggests that missed or not-rescheduled endoscopies in many cases were deemed unnecessary by the patients or that their prescription was not appropriate, in keeping with our recent analysis on open-access colonoscopies in the years before COVID-19 [14]. It is noteworthy to point out that at present we do not know how many outpatients failed to book an endoscopy during the lockdown and the post-lockdown period.

In the first months of the pandemic, the discontinuation of endoscopic activity bookable through the NHS led us and other colleagues from other centres to raise a warning regarding the possibility of missed or delayed diagnosis of colorectal cancer in more advanced stages $[15,16]$. Despite the fact that our findings are limited to a single Gastroenterology Unit and cannot be considered representative of the Italian endoscopic activity, the drop of EGD and CSPY during the first year of COVID-19 that we describe here reinforces the concern of undiagnosed gastrointestinal cancers. In keeping with our concern is a recent survey that reports a decrease in diagnosis of cancer involving the colon, pancreas, and stomach in Italy during the first year of COVID-19 [17]. This finding is in line with the reduction in the diagnosis of colorectal cancer in the United Kingdom [13] and of all cancers in the Netherlands [18]. The future impact of a delay in diagnosis of cancer has been estimated in different countries. In Hong Kong, it has been calculated that $4.6 \%$ and $6.4 \%$ of patients with gastric and colorectal cancer would have cancer stage upshifting because of a delayed diagnosis of 6 months [19]. In the United Kingdom, compared with pre-pandemic figures, it has been estimated that there has been a $15.3-16.6 \%$ increase in the number of deaths due to colorectal cancer, and a $5.8-6.0 \%$ in- 
crease of deaths due oesophageal cancer up to 5 years after diagnosis [20]. It has been hypothesised that the true impact, in terms of mortality, due to COVID-19-related disruption to cancer pathways will not be fully evident for 10 years or longer [21]. In a modelling study, it has been estimated that the number of lives lost increases according to diagnostic delays due to the inability to face the backlog of procedures postponed during COVID-19, the attributable lives lost being highest for colorectal cancer (number of lives lost: 296 for 1 month of delay - 2196 for 6 months of delay) [21]. The authors of such a modelling study conclude that only a prompt provision of additional capacity to address the backlog of diagnostics will minimise deaths as a result of diagnostic delays that could add to those predicted due to expected presentational delays [21]. In keeping with our previous suggestion [15], investment in expansion of capacity for NHS diagnostics and treatment is a priority if cancer services are to become more resilient to future extrinsic disruption, which could include additional waves of COVID-19 [21]. Therefore, resumption and maintenance of an effective endoscopic activity are crucial for containing the mortality and improve the prognosis of Gl disorders, primarily cancers, and there is a great effort to define the best strategies to make it happen [22].

The impact of COVID-19 on ERCP and EUS was less pronounced because in most cases they were nondeferrable. However, the consistent reduction of ERCP and EUS during the lockdown suggests that also patients with diseases involving the biliopancreatic district waited for as long as possible before entering the hospital when it was no longer postponable, similarly to patients with ischaemic heart disease [23]. This would explain the significant rebound of ERCPs during the post-lockdown period. In contrast, we observed a great reduction of ERCP and EUS in the period from November 2020 to January 2021, when the effects of the second wave of the epidemic were most evident. This fall could be the result of less patient access to the hospital, consistent with what happened in the lockdown period. Again, in February-March 2021, ERCP showed a significant rebound. It is tempting to speculate that we will continue to have similar fluctuations until we control the pandemic. At the time this paper was drafted, COVID-19 vaccination has commenced worldwide, and this will speed up the recovery of digestive endoscopy. Indeed, the efficacy of vaccination on COVID-19 diffusion depends on the development of mutant SARSCoV-2 strains with different epidemiological impact and the occurrence of vaccine-related side effects that may slow down the immunization process. It is conceivable that we will have to face COVID-19 for a long time to come. Therefore, the reopening of endoscopic services depends on a balance between the need to continue to protect health workers and the availability of economic resources and personnel, which may vary according to the hospitalizations of COVID-19 patients. Throughout the pandemic, the shortage of anaesthesiologists, especially during the 2 waves, strongly influenced biliopancreatic endoscopic procedures. To date, the possibility to receive conscious or deep sedation during an endoscopic examination varies between different centres and countries due to different guidelines and recommendations [24]. Indeed, ERCP and EUS-FNA, and other interventional procedures like EGD-PEG, require anaesthesia due to their invasiveness and the need for absolute immobility of the patient to increase the chances of success and reduce the risk of complications. During both waves of the pandemic, almost all our anaesthesiologists had been called to play a tireless endeavour for supporting the vital functions of COVID-19 patients in critical condition, and often they could not guarantee any other activity except the operating room. In many cases, we waited a whole day for the availability of an anaesthesiologist to undertake an ERCP or an EGD-PEG, and sometimes the intervention was postponed. In keeping with our experience, a French study [25] reported that $71 \%$ of anaesthesiologists dedicated to endoscopy had been requisitioned to assist COVID-19 patients during the first outbreak wave. This evidence confirms that, like other interventional disciplines, such as the Haemodynamic Unit and Radiology Unit, the inclusion of an anaesthesiologist in the team of an Endoscopy Unit is necessary for appropriate care in frail patients and for performing complex endoscopic procedures, as largely discussed in the literature [26, 27].

A way to ensure endoscopic activity during the persisting COVID-19 outbreak and to optimize the resources of the public health system for high-quality healthcare in the future is the promotion of interhospital collaboration between physicians with different skills and levels of experience [28-30]. Our collaboration network with neighbouring hospitals, which we have been able to put in place, has revealed to be crucial for delivering in a short time a mandatory procedure with primary benefit to the patient and a secondary saving effect thanks to the reduction in hospitalization times and related costs.

Finally, a strategy for preventing SARS-CoV-2 spread, including not only the use of personal protection equipment, was, and still is, adopted in our Endoscopy Unit, like in most other Italian endoscopy centres [3, 4]. Such 'countermeasures' have proven to be appropriate because only a few members of healthcare staff contracted the infection, and mainly due to external reasons. 
Despite the fact that all staff in our Endoscopy Unit have received a vaccine against COVID-19, we will continue to pay great attention to complying with all safety procedures and wearing all PPE.

\section{Conclusions}

The endoscopic activity in our Gastroenterology centre decreased significantly during the lockdown but also throughout the first year of the COVID-19 pandemic. The greatest impact was on elective EGDS and CSPY in outpatients, while nondeferrable ERCP were less affected. The pandemic-related limitations and the backlog of endoscopic procedures represent important reasons for the increased risk or delayed diagnosis of GI cancers. Urgent intervention by NHS stakeholders is necessary for the rationalization of resources and the restoration of digestive endoscopic activity to the pre-COVID-19 level.

\section{Acknowledgments}

We would like to thank all our nursing staff and dedicate this study to the nurses who were relocated to the COVID-19 wards: Alessandra Colonna, Catia Fiocco, Antonella Giaquinto, Viviana Pignatiello, Mezia Sibilia, Stefania Spinelli, and Cesare Vittori.

\section{Conflict of interest}

The authors declare no conflict of interest.

\section{References}

1. https://www.euro.who.int/en/health-topics/health-emergencies/coronavirus-covid-19/news/news/2020/3/who-announces-covid-19-outbreak-a-pandemic (Accessed March 24th 2021).

2. World Health Organization Rational Use of personal protective equipment for coronavirus disease (COVID-19): interim guidance, 27 February 2020, Geneva: World Health Organization; 2020. https://extranet.who.int/iris/restricted/handle/ 10665/331215.

3. Repici A, Pace F, Gabbiadini R, et al. Endoscopy units and the coronavirus disease 2019 outbreak: a multicenter experience from Italy. Gastroenterology 2020; 159: 363-6.e3.

4. Mirante VG, Mazzi G, Bevivino G, et al. How an endoscopy unit changed and was partially converted into an ICU during COVID-19 emergency in a tertiary referral hospital of Northern Italy. Dig Liver Dis 2021; 53: 1-3.

5. Maida M, Sferrazza S, Savarino E, et al. Impact of the COVID-19 pandemic on Gastroenterology Divisions in Italy: a national survey. Dig Liver Dis 2020; 52: 808-15.

6. Salerno R, Conti CB, De Silvestri A, et al.; The ITALIAN URGENT ENDOSCOPY - COVID-19 Working Group. The impact of covid-19 pandemic on urgent endoscopy in Italy: a nation-wide multicenter study. Scand J Gastroenterol 2020; 55: 870-6.

7. Gralnek IM, Hassan C, Beilenhoff U, et al. ESGE and ESGENA Position Statement on gastrointestinal endoscopy and
COVID-19: An update on guidance during the post-lockdown phase and selected results from a membership survey. Endoscopy 2020; 52: 819-98.

8. Gralnek IM, Hassan C, Beilenhoff U, et al. ESGE and ESGENA Position Statement on gastrointestinal endoscopy and the COVID-19 pandemic. Endoscopy 2020; 52: 483-90.

9. Hassan C, Wysocki PT, Fuccio L, et al. Endoscopic surveillance after surgical or endoscopic resection for colorectal cancer: European Society of Gastrointestinal Endoscopy (ESGE) and European Society of Digestive Oncology (ESDO) Guideline. Endoscopy 2019; 51: 266-77.

10. Hassan C, Antonelli G, Dumonceau JM, et al. Post-polypectomy colonoscopy surveillance: European Society of Gastrointestinal Endoscopy (ESGE) Guideline - Update 2020 Endoscopy 2020; 52: 687-700.

11. https://www.buonepratichesicurezzasanita.it/index.php/ component/judirectory/13-soluzioni-organizzative-emergenziali-per-la-gestione-dei-pazienti-covid/143-istruzione-operativa-per-la-gestione-di-pazienti-ricoverati-da-sottoporre-adesami-endoscopici-durante-la-pandemia-da-sars-cov-2-virus? Itemid=101.

12. Schmiderer A, Schwaighofer $\mathrm{H}$, Niederreiter $\mathrm{L}$, et al. Decline in acute upper gastrointestinal bleeding during COVID-19 pandemic after initiation of lockdown in Austria. Endoscopy 2020; 52: 1036-8.

13. Rutter MD, Brookes M, Lee TJ, et al. Impact of the COVID-19 pandemic on UK endoscopic activity and cancer detection: a National Endoscopy Database Analysis. Gut 2021; 70: 537-43.

14. Del Vecchio Blanco G, Dwairi R, Giannelli M, et al. Clinical care pathway program versus open-access system: a study on appropriateness, quality, and efficiency in the delivery of colonoscopy in the colorectal cancer. Intern Emerg Med 2021; 16: 1197-206.

15. Del Vecchio Blanco G, Calabrese E, Biancone L, et al. The impact of COVID-19 pandemic in the colorectal cancer prevention. Int J Colorectal Dis 2020; 4: 1-4.

16. Gralnek IM, Hassan C, Dinis-Ribeiro M. COVID-19 and endoscopy: implications for healthcare and digestive cancer screening Nat Rev Gastroenterol Hepatol 2020; 17: 444-6.

17. Buscarini E, Benedetti A, Monica F, et al. Changes in digestive cancer diagnosis during the SARS-CoV-2 pandemic in Italy: a nationwide survey. Dig Liver Dis 2021; 53: P682-8.

18. Dinmohamed AG, Visser O, Verhoeven RHA, et al. Fewer cancer diagnoses during the COVID-19 epidemic in the Netherlands. Lancet Oncol 2020; 21: 750-1.

19. Lui TKL, Leung K, Guo CG, et al. Impacts of the Coronavirus 2019 pandemic on gastrointestinal endoscopy volume and diagnosis of gastric and colorectal cancers: a population-based study. Gastroenterology 2020; 159: 1164-6.

20. Maringe C, Spicer J, Morris M, et al. The impact of the COVID-19 pandemic on cancer deaths due to delays in diagnosis in England, UK: a national, population-based, modelling study. Lancet Oncol 2020; 21: 1023-34.

21. Sud A, Torr B, Jones ME, et al. Effect of delays in the 2-weekwait cancer referral pathway during the COVID-19 pandemic on cancer survival in the UK: a modelling study. Lancet Oncol 2020; 21: 1035-44. 
22. Antonelli G, Karsensten JG, Bhat P, et al. Resuming endoscopy during COVID-19 pandemic: ESGE, WEO and WGO Joint Cascade Guideline for Resource Limited Settings. Endosc Int Open 2021; 9: E543-51.

23. De Rosa S, Spaccarotella C, Basso C, et al. Reduction of hospitalizations for myocardial infarction in Italy in the COVID-19 era. Eur Heart J 2020; 41: 2083-8.

24. Dossa F, Megetto O, Yakubu M, et al. Sedation practices for routine gastrointestinal endoscopy: a systematic review of recommendations. BMC Gastroenterol 2021; 21: 22.

25. Belle A, Barret M, Bernardini D, et al. Impact of the COVID-19 pandemic on gastrointestinal endoscopy activity in France. Endoscopy 2020; 52: 1111-5.

26. ASGE Standards of Practice Committee; Early DS, Lightdale JR, et al. Guidelines for sedation and anesthesia in GI endoscopy. Gastrointest Endosc 2018; 87: 327-37.

27. Sidhu R, Turnbull D, Newton M, et al. Deep sedation and anaesthesia in complex gastrointestinal endoscopy: a joint position statement endorsed by the British Society of Gastroenterology (BSG), Joint Advisory Group (JAG) and Royal College of Anaesthetists (RCoA). Frontline Gastroenterol. 2019; 10: 141-7.

28. Mascia D, Di Vincenzo F. Understanding hospital performance: the role of network ties and patterns of competition. Health Care Manage Rev 2011; 36: 327-37.

29. Uddin S, Hossain L, Kelaher M. Effect of physician collaboration network on hospitalization cost and readmission rate Eur J Public Health 2012; 22: 629-33.

30. Mascia D, Angeli F, Di Vincenzo F. Effect of hospital referral networks on patient readmissions. Soc Sci Med 2015; 132 113-21.

Received: 10.06 .2021

Accepted: 3.11 .2021 